\title{
Bi-Bazilevič functions based on the Mittag-Leffler-type Borel distribution associated with Legendre polynomials
}

\author{
Sheza. M. El-Deeb ${ }^{\mathrm{a}, \mathrm{b}, *}$, G. Murugusundaramoorthy ${ }^{\mathrm{c}}$, Alhanouf Alburaikan ${ }^{\mathrm{a}}$ \\ ${ }^{a}$ Department of Mathematics, College of Science and Arts, Al-Badaya, Qassim University, Buraidah, Saudi Arabia. \\ ${ }^{b}$ Department of Mathematics, Faculty of Science, Damietta University, New Damietta 34517, Egypt. \\ ${ }^{c}$ Department of Mathematics, School of Advanced Sciences, Vellore Institute Technology University, Vellore-632014, India.
}

\begin{abstract}
In this paper, using the Mittag-Leffler-type Borel distribution, the authors introduce a new class of bi-Bazilevic functions defined in the open unit disc associated with Legendre polynomials, we find estimates for the general Taylor-Maclaurin coefficients of the functions in the subclass introduced, and the Fekete-Szego problem is solved.
\end{abstract}

Keywords: Bi-Bazilevic functions, coefficient estimates, Mittag-Leffler-type, Borel distribution, Legendre polynomials. 2020 MSC: 30C45, 30C50.

(C)2022 All rights reserved.

\section{Introduction, definitions, and preliminaries}

Let $\mathcal{A}$ denote the class of analytic functions of the form

$$
f(z):=z+\sum_{k=2}^{\infty} a_{k} z^{k}, \quad z \in \Delta:=\{z \in \mathbb{C}:|z|<1\}
$$

and let $\mathcal{S} \subset \mathcal{A}$ consisting on functions that are univalent in $\Delta$.

The convolution or the Hadamard product of two functions $f_{1}, f_{2} \in \mathcal{A}$ is denoted by $f_{1} * f_{2}$ and is defined as follows:

$$
\left(f_{1} * f_{2}\right)(z):=z+\sum_{k=2}^{\infty} a_{k, 1} a_{k, 2} z^{k}=:\left(f_{2} * f_{1}\right)(z),
$$

where

$$
f_{i}(z)=z+\sum_{k=2}^{\infty} a_{k, i} z^{k}, \quad i=1,2 .
$$

For $0 \leqslant \gamma<1$, and $f \in \mathcal{S}$ is as assumed in (1.1), then

\footnotetext{
*Corresponding author

Email addresses: shezaeldeeb@yahoo.com (Sheza. M. El-Deeb), gmsmoorthy@yahoo.com (G. Murugusundaramoorthy),

a. albrikan@qu.edu.sa (Alhanouf Alburaikan)
}

doi: $10.22436 /$ jmcs.024.03.05

Received: 2020-12-18 Revised: 2020-12-31 Accepted: 2021-01-18 
1. $f$ is said to be starlike of order $\gamma$ if $\mathcal{S}^{*}(\gamma)=\left\{f \in \mathcal{A}: \mathfrak{R}\left(\frac{z f^{\prime}(z)}{f(z)}\right)>\gamma, \quad z \in \Delta\right\}$;

2. $f$ is said to be convex of order $\gamma$ if $\mathcal{K}(\gamma)=\left\{f \in \mathcal{A}: \mathfrak{R}\left(1+\frac{z f^{\prime \prime}(z)}{f^{\prime}(z)}\right)>\gamma, \quad z \in \Delta\right\}$.

An analytic function $F$ is subordinate to another analytic function $G$, written as follows:

$$
\mathrm{F}(z) \prec \mathrm{G}(z), \quad(z \in \Delta),
$$

provided that there exists an analytic function (that is, Schwarz function) $\omega(z)$ defined on $\Delta$ with

$$
\omega(0)=0 \quad \text { and } \quad|\omega(z)|<1, \quad(z \in \Delta)
$$

such that (see, for details, [27])

$$
\mathrm{F}(z)=\mathrm{G}(\omega(z)), \quad(z \in \Delta) .
$$

Ma and Minda [26] unified various subclasses of starlike and convex functions for which either of the functions

$$
\frac{z f^{\prime}(z)}{f(z)}, \quad \text { and } \quad 1+\frac{z f^{\prime \prime}(z)}{f^{\prime}(z)}
$$

is subordinate to a more general superordinate function. For this purpose, they considered an analytic function $\phi$, with

$$
\mathfrak{R}(\phi(z))>0, \quad(z \in \Delta), \quad \phi(0)=1, \quad \text { and } \quad \phi^{\prime}(0)>0,
$$

which maps $\Delta$ onto a region starlike with respect to 1 and symmetric with respect to the real axis. The class of Ma-Minda starlike functions and Ma-Minda convex functions in $\Delta$ consists of functions $f \in \mathcal{A}$ satisfying the following subordination condition:

$$
\frac{z f^{\prime}(z)}{f(z)} \prec \phi(z), \quad \text { and } \quad 1+\frac{z f^{\prime \prime}(z)}{f^{\prime}(z)} \prec \phi(z),
$$

$z \in \Delta$ respectively. Such a function has a series expansion of the following form:

$$
\phi(z)=1+c_{1} z+c_{2} z^{2}+c_{3} z^{3}+\cdots, \quad\left(c_{1}>0 ; z \in \Delta\right) .
$$

\subsection{Bi-univalent functions}

It is well known that every function $f \in \mathcal{S}$ has an inverse $f^{-1}$, defined by

$$
f^{-1}(f(z))=z, \quad(z \in \Delta) \quad \text { and } \quad f\left(f^{-1}(w)\right)=w, \quad\left(|w|<r_{0}(f) ; r_{0}(f) \geqq \frac{1}{4}\right),
$$

where

$$
g(w)=f^{-1}(w)=w-a_{2} w^{2}+\left(2 a_{2}^{2}-a_{3}\right) w^{3}-\left(5 a_{2}^{3}-5 a_{2} a_{3}+a_{4}\right) w^{4}+\cdots .
$$

A function $\mathrm{f} \in \mathcal{A}$ is said to be bi-univalent in $\Delta$ if both $\mathrm{f}(z)$ and $\mathrm{f}^{-1}(z)$ are univalent in $\Delta$. Let $\Sigma$ denote the class of bi-univalent functions in $\Delta$ given by (1.1). Note that the functions $f_{1}(z)=\frac{z}{1-z}, f_{2}(z)=$ $\frac{1}{2} \log \frac{1+z}{1-z}, f_{3}(z)=-\log (1-z)$, with their corresponding inverses $f_{1}^{-1}(w)=\frac{w}{1+w^{\prime}}, f_{2}^{-1}(w)=\frac{e^{2 w}-1}{e^{2 w}+1}$, $f_{3}^{-1}(w)=\frac{e^{w}-1}{e^{w}}$, are elements of $\Sigma$. The class of analytic bi-univalent functions was first introduced by Lewin [24], where it was proved that $\left|\mathrm{a}_{2}\right|<1.51$. Lately, especially after its revival by Srivastava et al. [37], there has been triggering interest in the study of the bi-univalent function class $\Sigma$ leading to nonsharp coefficient estimates on the first two Taylor-Maclaurin coefficients $\left|a_{2}\right|$ and $\left|a_{3}\right|$ in (1.1). However, the coefficient problem for each of the following Taylor-Maclaurin coefficients:

$$
\left|a_{k}\right|, \quad(k \in \mathbb{N} \backslash\{1,2\}),
$$


is still an open problem (see [6-8, 24, 31, 39]). Motivated largely by (and following the work of) Srivastava et al. [37], many researchers (see, for example, [9, 11, 16, 18, 19, 25, 36]) have recently introduced and investigated several interesting subclasses of the bi-univalent function class $\Sigma$ and they have found nonsharp estimates on the corresponding first two Taylor-Maclaurin coefficients $\left|a_{2}\right|$ and $\left|a_{3}\right|$.

Several authors have discussed various subfamilies of the well-known Bazilevič functions (see, for details, [10, 35]; see also [1-3, 20, 21, 32, 34, 35, 41]) of type $\lambda$ from various viewpoints such as the perspective of convexity, inclusion theorems, radius of starlikeness and convexity, boundary rotational problems, subordination relationships, and so on. It is interesting to note in this connection that the earlier investigations on the subject do not seem to have addressed the problems involving coefficient inequalities and coefficient bounds for these subfamilies of Bazilevič type functions especially when the parameter $\lambda$ is greater than $1(\lambda \in \mathbb{R})$. Thus, motivated primarily by the recent work of Deniz [11](see $[30,38])$, we introduce here a new subfamily of Bazilevič type functions belonging to the function class $\Sigma$ involving the Borel distribution operator associated with Mittag-Leffler function. For this new subfamily of Bazilevič type functions, we find estimates on the Taylor-Maclaurin coefficients $\left|a_{2}\right|$ and $\left|a_{3}\right|$. Several closely-related function classes are also considered and relevant connections to earlier known results are pointed out.

\subsection{Mittag-Leffler function and Borel distribution:}

The study of operators plays an important rôle in geometric function theory in complex analysis and its related fields. Many derivative and integral operators can be written in terms of convolution of certain analytic functions. It is observed that this formalism brings an ease in further mathematical exploration and also helps to better understand the geometric properties of such operators.

Let $\mathbf{E}_{\alpha}(z)$ and $\mathbf{E}_{\alpha, \beta}(z)$ be functions defined by

$$
\mathbf{E}_{\alpha}(z)=\sum_{k=0}^{\infty} \frac{z^{k}}{\Gamma(\alpha k+1)}, \quad(z \in \mathbb{C}, \Re(\alpha)>0)
$$

and

$$
\mathbf{E}_{\alpha, \beta}(z)=\frac{1}{\Gamma(\beta)}+\sum_{k=1}^{\infty} \frac{z^{k}}{\Gamma(\alpha k+\beta)}, \quad(\alpha, \beta \in \mathbb{C}, \mathfrak{R}(\alpha)>0, \mathfrak{R}(\beta)>0) .
$$

It can be written in other form

$$
\mathbf{E}_{\alpha, \beta}(z)=\frac{1}{\Gamma(\beta)}+\sum_{k=2}^{\infty} \frac{z^{k-1}}{\Gamma(\alpha(k-1)+\beta)}, \quad(\alpha, \beta \in \mathbb{C}, \mathfrak{R}(\alpha)>0, \mathfrak{R}(\beta)>0) .
$$

The function $\mathbf{E}_{\alpha}(z)$ was introduced by Mittag-Leffler [28] and is, therefore, known as the Mittag-Leffler function. A more general function $E_{\alpha, \beta}$ generalizing $E_{\alpha}(z)$ was introduced by Wiman [42] and defined by

$$
\mathbf{E}_{\alpha, \beta}(z)=\sum_{k=0}^{\infty} \frac{z^{k}}{\Gamma(\alpha \mathrm{k}+\beta)}, \quad(z, \alpha, \beta \in \mathbb{C}, \mathfrak{R}(\alpha)>0, \mathfrak{R}(\beta)>0) .
$$

Observe that the function $\mathbf{E}_{\alpha, \beta}$ contains many well-known functions as its special case, for example, $\mathbf{E}_{1,1}(z)=e^{z}, \mathbf{E}_{1,2}(z)=\frac{e^{z}-1}{z}, \mathbf{E}_{2,1}\left(z^{2}\right)=\cosh z, \mathbf{E}_{2,1}\left(-z^{2}\right)=\cos z, \mathbf{E}_{2,2}\left(z^{2}\right)=\frac{\sinh z}{z}, \mathbf{E}_{2,2}\left(-z^{2}\right)=\frac{\sin z}{z}$, $\mathbf{E}_{4}(z)=\frac{1}{2}\left[\cos z^{1 / 4}+\cosh z^{1 / 4}\right]$, and $\mathbf{E}_{3}(z)=\frac{1}{2}\left[e^{z^{1 / 3}}+2 e^{-\frac{1}{2} z^{1 / 3}} \cos \left(\frac{\sqrt{3}}{2} z^{1 / 3}\right)\right]$. The Mittag-Leffler function arises naturally in the solution of fractional order differential and integral equations, and especially in the investigations of fractional generalization of kinetic equation, random walks, Lévy flights, superdiffusive transport and in the study of complex systems. Several properties of Mittag-Leffler function and generalized Mittag-Leffler function can be found, e.g., in [4, 5, 14, 15, 17, 22, 33]. Observe that MittagLeffler function $\mathbf{E}_{\alpha, \beta}(z)$ does not belong to the family $\mathcal{A}$. Thus, it is natural to consider the following 
normalization of Mittag-Leffler functions as below:

$$
\mathrm{E}_{\alpha, \beta}(z)=z \Gamma(\beta) \mathbf{E}_{\alpha, \beta}(z)=z+\sum_{\mathrm{k}=2}^{\infty} \frac{\Gamma(\beta)}{\Gamma(\alpha(\mathrm{k}-1)+\beta)} z^{\mathrm{k}},
$$

it holds for complex parameters $\alpha, \beta$ and $z \in \mathbb{C}$. In this paper, we shall restrict our attention to the case of real-valued $\alpha, \beta$ and $z \in \Delta$.

A discrete random variable $x$ is said to have a Borel distribution if it takes the values $1,2,3, \ldots$ with the probabilities $\frac{\mathrm{e}^{-\lambda}}{1 !}, \frac{2 \lambda e^{-2 \lambda}}{2 !}, \frac{9 \lambda^{2} \mathrm{e}^{-3 \lambda}}{3 !}, \ldots$, respectively, where $\lambda$ is called the parameter.

Very recently, Wanas and Khuttar [40] introduced the Borel distribution (BD) whose probability mass function is

$$
P(x=\rho)=\frac{(\rho \lambda)^{\rho-1} e^{-\lambda \rho}}{\rho !}, \quad \rho=1,2,3, \ldots
$$

Wanas and Khuttar introduced a series $\mathcal{M}(\lambda ; z)$ whose coefficients are probabilities of the Borel distribution (BD)

$$
\mathcal{M}(\lambda ; z)=z+\sum_{k=2}^{\infty} \frac{[\lambda(k-1)]^{k-2} e^{-\lambda(k-1)}}{(k-1) !} z^{k},(0<\lambda \leqslant 1),
$$

In [29], the authors defined the Mittag-Leffler-type Borel distribution as follows:

$$
\mathcal{P}(\lambda, \alpha, \beta ; \rho)=\frac{(\lambda \rho)^{\rho-1}}{\mathrm{E}_{\alpha, \beta}(\lambda \rho) \Gamma(\alpha \rho+\beta)}, \quad \rho=0,1,2, \ldots,
$$

where

$$
\mathrm{E}_{\alpha, \beta}(z)=\sum_{k=0}^{\infty} \frac{z^{\mathrm{k}}}{\Gamma(\alpha \mathrm{k}+\beta)}, \quad(\alpha, \beta \in \mathbb{C}, \mathfrak{R}(\alpha)>0, \mathfrak{R}(\beta)>0) .
$$

Thus by using (1.3) and (1.4) and by convolution operator, we define the Mittag-Leffler-type Borel distribution series as below

$$
\mathcal{B}(\lambda, \alpha, \beta)(z)=z+\sum_{k=2}^{\infty} \frac{(\lambda(k-1)) ![\lambda(k-1)]^{k-2} e^{-\lambda(k-1)}}{(k-1) ! E_{\alpha, \beta}(\lambda(k-1)) \Gamma(\alpha(k-1)+\beta)} z^{k},(0<\lambda \leqslant 1) .
$$

Further, by the convolution operator we define

$$
\begin{aligned}
\mathcal{B}(\lambda, \alpha, \beta) f(z) & =\mathcal{B}(\lambda, \alpha, \beta)(z) * f(z) \\
& =z+\sum_{k=2}^{\infty} \frac{(\lambda(k-1)) ![\lambda(k-1)]^{k-2} e^{-\lambda(k-1)}}{(k-1) ! \mathrm{E}_{\alpha, \beta}(\lambda(k-1)) \Gamma(\alpha(k-1)+\beta)} a_{k} z^{k} \\
& =z+\sum_{k=2}^{\infty} \phi_{k} a_{k} z^{k}, \quad(\alpha, \beta \in \mathbb{C}, \mathfrak{R}(\alpha)>0, \mathfrak{R}(\beta)>0,0<\lambda \leqslant 1),
\end{aligned}
$$

where

$$
\phi_{k}=\frac{(\lambda(k-1)) ![\lambda(k-1)]^{k-2} e^{-\lambda(k-1)}}{(k-1) ! \mathrm{E}_{\alpha, \beta}(\lambda(k-1)) \Gamma(\alpha(k-1)+\beta)} .
$$

\subsection{Bi-Bazilevič functions associated with Legendre polynomials}

Legendre polynomials, which are exceptional cases of Legendre functions, are familiarized in 1784 by the French mathematician Legendre (1752-1833). Legendre functions are a vital and important in problems 
including spherical coordinates. As well, the Legendre polynomials, $P_{k}(x),(|x|<1)$, are designated via the following generating function (see $[12,23])$ :

$$
\mathrm{G}(x, z)=\frac{1}{\sqrt{1-2 x z+z^{2}}}=\sum_{\mathrm{k}=0}^{\infty} \mathrm{P}_{\mathrm{k}}(\mathrm{x}) z^{\mathrm{k}} \text {. }
$$

Legendre polynomials are the everywhere regular solutions of Legendrâe differential equation that we can write as follows:

$$
\left(1-x^{2}\right) \frac{d^{2}}{d x^{2}} P_{k}(x)-2 x \frac{d}{d x} P_{k}(x)+m P_{k}(x)=0,
$$

where $m=k(k+1)$ and $k=0,1,2, \ldots$ Taking $x=1$ in (1.6) and by using geometric series, we see that $P_{k}(1)=1$, so that the Legendre polynomials are normalized. Thus Let $G(x, z)$ denote the class of analytic functions on $\Delta$ which are normalized by the conditions $G(x, 0)=0$ and $G^{\prime}(x, 0)=1$.

Definition 1.1. Let $P_{k}(x)$ is Legendre polynomials of the first kind of order $k=0,1,2, \ldots$, the recurrence formula is

with

$$
P_{k+1}(x)=\frac{2 k+1}{k+1} x P_{k}(x)-\frac{k}{k+1} P_{k-1}(x),
$$

$$
P_{0}(x)=1 \quad \text { and } \quad P_{1}(x)=x .
$$

Motivated by the earlier work of Srivastava et al. [38], we define the following subclass of functions $\mathcal{M}_{\Sigma}^{\lambda, \alpha, \beta}(\delta, \sigma ; \chi)$ related with Mittag-Leffler-type Borel distribution subordinating with Legendre polynomials as in Definition 1.2. We obtain the estimates on the initial Taylor-Maclaurin coefficients and the Fekete-Szegö inequalities for this subclass of the bi-univalent function class $\Sigma$. We also give results for new function classes of the bi-univalent function class which we introduce here.

Definition $1.2([13,29])$. Let the function $G(x, z)$ is given by

$$
\mathrm{G}(x, z)=1+\sum_{k=1}^{\infty} \mathrm{P}_{k}(x) z^{k}, \quad(z \in \Delta) .
$$

For $\alpha, \beta \in \mathbb{C}, \mathfrak{R}(\alpha)>0, \mathfrak{R}(\beta)>0,0<\lambda \leqslant 1$, a function $\mathrm{f} \in \Sigma$ given by (1.1) is said to be in the class $\mathcal{M}_{\Sigma}^{\lambda, \alpha, \beta}(\delta, \sigma ; x)$ if the following conditions are satisfied:

$$
e^{i \delta}\left(\frac{z^{1-\sigma}(\mathcal{B}(\lambda, \alpha, \beta) f(z))^{\prime}}{[\mathcal{B}(\lambda, \alpha, \beta) f(z)]^{1-\sigma}}\right) \prec G(x, z) \cos \delta+i \sin \delta
$$

and

$$
e^{i \delta}\left(\frac{w^{1-\sigma}(\mathcal{B}(\lambda, \alpha, \beta) g(w))^{\prime}}{[\mathcal{B}(\lambda, \alpha, \beta) g(w)]^{1-\sigma}}\right) \prec G(x, w) \cos \delta+i \sin \delta
$$

where

$$
\delta \in\left(-\frac{\pi}{2}, \frac{\pi}{2}\right), \quad \sigma \geqslant 0, \quad \text { and } \quad z, w \in \Delta,
$$

and the function $\mathrm{g}$ is given by (1.2).

Remark 1.3. In the Definition 1.2, by taking $\delta=0$ we define the following new class.

1. A function $f \in \Sigma$ given by (1.1) is said to be in the class $\mathcal{B}_{\Sigma}^{\lambda, \alpha, \beta}(\sigma ; x)$ if the following conditions are satisfied:

$$
\left(\frac{z^{1-\sigma}(\mathcal{B}(\lambda, \alpha, \beta) f(z))^{\prime}}{[\mathcal{B}(\lambda, \alpha, \beta) f(z)]^{1-\sigma}}\right) \prec G(x, z) \quad \text { and } \quad\left(\frac{w^{1-\sigma}(\mathcal{B}(\lambda, \alpha, \beta) g(w))^{\prime}}{[\mathcal{B}(\lambda, \alpha, \beta) g(w)]^{1-\sigma}}\right) \prec G(x, w),
$$

where $\sigma \geqslant 0 ; z, w \in \Delta$ and the function $g$ is given by (1.2). A function $f \in \Sigma$ given by (1.1) is said to be in the class. 
2. $\mathcal{M}_{\Sigma}^{\lambda, \alpha, \beta}(\delta, 0 ; x) \equiv \mathcal{S}_{\Sigma}^{\lambda, \alpha, \beta}(\delta ; x)$ if the following conditions are satisfied:

$$
e^{i \delta}\left(\frac{z(\mathcal{B}(\lambda, \alpha, \beta) f(z))^{\prime}}{[\mathcal{B}(\lambda, \alpha, \beta) f(z)]}\right) \prec G(x, z) \cos \delta+i \sin \delta
$$

and

$$
e^{i \delta}\left(\frac{w(\mathcal{B}(\lambda, \alpha, \beta) g(w))^{\prime}}{[\mathcal{B}(\lambda, \alpha, \beta) g(w)]}\right) \prec G(x, w) \cos \delta+i \sin \delta,
$$

where $z, w \in \Delta$ and the function $g$ is given by (1.2).

3. $\mathcal{M}_{\Sigma}^{\lambda, \alpha, \beta}(\delta, 1 ; x) \equiv \mathcal{R}_{\Sigma}^{\lambda, \alpha, \beta}(\delta ; x)$ if the following conditions are satisfied:

$$
e^{i \delta}\left((\mathcal{B}(\lambda, \alpha, \beta) f(z))^{\prime}\right) \prec G(x, z) \cos \delta+i \sin \delta
$$

and

$$
e^{i \delta}\left((\mathcal{B}(\lambda, \alpha, \beta) g(w))^{\prime}\right) \prec G(x, w) \cos \delta+i \sin \delta,
$$

where $z, w \in \Delta$ and the function $g$ is given by (1.2).

\section{Coefficient estimates}

For our study we restrict our attention to the case of real-valued $\alpha, \beta$ and let

$$
\delta \in\left(-\frac{\pi}{2}, \frac{\pi}{2}\right), \sigma \geqslant 0, \alpha, \beta \in \mathbb{C}, \mathfrak{R}(\alpha)>0, \mathfrak{R}(\beta)>0,0<\lambda \leqslant 1,
$$

unless otherwise stated. We introduce a bound for the initial coefficients of functions in $\mathcal{M}_{\Sigma}^{\lambda, \alpha, \beta}(\delta, \sigma ; \chi)$.

The following Lemma will be needed to prove our results.

Lemma 2.1 ([10]). If $w(z)=c_{1} z+c_{2} z^{2}+c_{3} z^{3}+\cdots, c_{1} \neq 0$ is analytic and satisfies $|w(z)|<1$ on the unit disk $\Delta$, then for each $0<\mathrm{r}<1,\left|w^{\prime}(0)\right|<1$ and $\left|w\left(r e^{i \delta}\right)\right|<1$ unless $w(z)=r e^{i \delta}$ for some real number $\delta$.

Theorem 2.2. Let $\mathrm{f}(z)$ as assumed in (1.1) and $\mathrm{f} \in \mathcal{M}_{\Sigma}^{\lambda, \alpha, \beta}(\delta, \sigma ; x)$. Then

$$
\begin{aligned}
& \left|a_{2}\right| \leqq \frac{|x \sqrt{2 x}| \cos ^{2} \delta}{\sqrt{x^{2} \cos \delta\left[(\sigma-1)(\sigma+2) \phi_{2}^{2}+2(\sigma+2) \phi_{3}\right]-\left(3 x^{2}-1\right)(\sigma+1)^{2} e^{i \delta} \phi_{2}^{2}}} \\
& \left|a_{3}\right| \leqq \frac{|x| \cos \delta}{(\sigma+2) \phi_{3}}+\left(\frac{x \cos \delta}{(\sigma+1) \phi_{2}}\right)^{2}
\end{aligned}
$$

and

$$
\left|a_{4}\right| \leqslant \frac{5 x^{2} \cos ^{2} \delta}{2(\sigma+1)(\sigma+2) \phi_{2} \phi_{3}}+\frac{5 x^{3} \cos ^{3} \delta}{2(\sigma+1)^{3} \phi_{2}^{3}}-\frac{N x^{3} \cos ^{3} \delta}{2(\sigma+1)^{3}(\sigma+3) \phi_{2}^{3} \phi_{4}}+\frac{\left(5 x^{3}+6 x^{2}-x-2\right) \cos \delta}{2(\sigma+3) \phi_{4}},
$$

where

$$
\mathrm{N}=5(\sigma+3) \phi_{4}+2(\sigma-1)(\sigma+3) \phi_{2} \phi_{3}+\frac{(\sigma-1)(\sigma-2)(\sigma+3)}{3} \phi_{2}^{3}
$$

$\delta \in\left(-\frac{\pi}{2}, \frac{\pi}{2}\right), \sigma \geqslant 0, \alpha, \beta \in \mathbb{C}, \mathfrak{R}(\alpha)>0, \mathfrak{R}(\beta)>0,0<\lambda \leqslant 1$ and the coefficients $\phi_{k}$ are as fixed in (1.5). 
Proof. It follows from (1.7) and (1.8) that

$$
e^{i \delta}\left(\frac{z^{1-\sigma}(\mathcal{B}(\lambda, \alpha, \beta) f(z))^{\prime}}{[\mathcal{B}(\lambda, \alpha, \beta) f(z)]^{1-\sigma}}\right) \prec G(x, z) \cos \delta+i \sin \delta
$$

and

$$
e^{i \delta}\left(\frac{z^{1-\sigma}(\mathcal{B}(\lambda, \alpha, \beta) g(w))^{\prime}}{[\mathcal{B}(\lambda, \alpha, \beta) g(w)]^{1-\sigma}}\right) \prec G(x, w) \cos \delta+i \sin \delta,
$$

where $G(x, z)$ and $G(x, w)$ are the generating function for Legendre polynomials with the following power series

$$
\mathrm{G}(\mathrm{x}, z)=1+\mathrm{P}_{1}(\mathrm{x}) z+\mathrm{P}_{2}(\mathrm{x}) z^{2}+\mathrm{P}_{3}(\mathrm{x}) z^{3}+\cdots=1+x z+\frac{1}{2}\left(3 x^{2}-1\right) z^{2}+\frac{1}{2}\left(5 x^{3}-3 x\right) z^{3}+\cdots, \quad(z \in \Delta)
$$

and

$$
\begin{aligned}
\mathrm{G}(\mathrm{x}, w) & =1+\mathrm{P}_{1}(\mathrm{x}) w+\mathrm{P}_{2}(\mathrm{x}) w^{2}+\mathrm{P}_{3}(\mathrm{x}) w^{3}+\cdots \\
& =1+x w+\frac{1}{2}\left(3 x^{2}-1\right) w^{2}+\frac{1}{2}\left(5 x^{3}-3 x\right) w^{3}+\cdots, \quad(w \in \Delta) .
\end{aligned}
$$

For some analytic $u$ and $v$ such that $u(0)=v(0)=0$ and $|u(z)|=\left|u_{1} z+u_{2} z^{2}+\cdots\right|<1, \quad|v(w)|=$ $\left|v_{1} w+v_{2} w^{2}+\cdots\right|<1$, for all $z, w \in \Delta$. It's well known that $\left|u_{k}\right| \leqslant 1,\left|v_{k}\right| \leqslant 1$ for every $k \in \mathbb{N}$. Now, equating the coefficients in (2.3) and (2.4), we get

$$
\begin{aligned}
e^{i \delta}(\sigma+1) \phi_{2} a_{2} & =u_{1} x \cos \delta, \\
e^{i \delta}\left(\frac{(\sigma-1)(\sigma+2)}{2} \phi_{2}^{2} a_{2}^{2}+(\sigma+2) \phi_{3} a_{3}\right) & =u_{2} x \cos \delta+\frac{1}{2} u_{1}^{2}\left(3 x^{2}-1\right) \cos \delta, \\
e^{i \delta}\left((\sigma+3) \phi_{4} a_{4}+(\sigma-1)(\sigma+3) \phi_{2} \phi_{3} a_{2} a_{3}\right. & \left.+\frac{(\sigma-1)(\sigma-2)(\sigma+3)}{6} \phi_{2}^{3} a_{2}^{3}\right) \\
=x u_{3} \cos \delta+\left(3 x^{2}-1\right) u_{1} u_{2} \cos \delta & +\frac{1}{2}\left(5 x^{3}-3 x\right) u_{1}^{3} \cos \delta, \\
-e^{i \delta}(\sigma+1) \phi_{2} a_{2} & =v_{1} x \cos \delta, \\
e^{i \delta}\left[\left(2(\sigma+2) \phi_{3}+\frac{(\sigma-1)(\sigma+2)}{2} \phi_{2}^{2}\right) a_{2}^{2}-(\sigma+2) \phi_{3} a_{3}\right] & =v_{2} x \cos \delta+\frac{1}{2} v_{1}^{2}\left(3 x^{2}-1\right) \cos \delta,
\end{aligned}
$$

and

$$
\begin{aligned}
& e^{i \delta}\left(\left[5(\sigma+3) \phi_{4}+(\sigma-1)(\sigma+3) \phi_{2} \phi_{3}\right] a_{2} a_{3}-\left[5(\sigma+3) \phi_{4}+2(\sigma-1)(\sigma+3) \phi_{2} \phi_{3}+\right.\right. \\
& \left.\left.\quad \frac{(\sigma-1)(\sigma-2)(\sigma+3)}{6} \phi_{2}^{3}\right] a_{2}^{3}-(\sigma+3) \phi_{4} a_{4}\right) \\
& =x v_{3} \cos \delta+\left(3 x^{2}-1\right) v_{1} v_{2} \cos \delta+\frac{1}{2}\left(5 x^{3}-3 x\right) v_{1}^{3} \cos \delta
\end{aligned}
$$

From (2.5) and (2.8), we find that

$$
a_{2}=\frac{u_{1} e^{-i \delta} x \cos \delta}{(\sigma+1) \phi_{2}}=-\frac{\nu_{1} e^{-i \delta} x \cos \delta}{(\sigma+1) \phi_{2}}
$$

which implies that

$$
\mathrm{u}_{1}=-\mathrm{v}_{1}
$$

and

$$
2 e^{2 i \delta}\left[(\sigma+1) \phi_{2} a_{2}\right]^{2}=\left(u_{1}^{2}+v_{1}^{2}\right) x^{2} \cos ^{2} \delta .
$$


Upon adding (2.6) and (2.9), if we make use of (2.11) and (2.12), we obtain

$$
e^{i \delta}\left[(\sigma-1)(\sigma+2) \phi_{2}^{2}+2(\sigma+2) \phi_{3}\right] a_{2}^{2}=\left(u_{2}+v_{2}\right) x \cos \delta+\frac{1}{2}\left(3 x^{2}-1\right) \cos \delta\left(u_{1}^{2}+v_{1}^{2}\right),
$$

which yields

$$
a_{2}^{2}=\frac{\left(u_{2}+v_{2}\right) x^{3} e^{-i \delta} \cos ^{2} \delta}{x^{2} \cos \delta\left[(\sigma-1)(\sigma+2) \phi_{2}^{2}+2(\sigma+2) \phi_{3}\right]-\left(3 x^{2}-1\right)(\sigma+1)^{2} e^{i \delta} \phi_{2}^{2}} .
$$

Applying Lemma 2.1 for the coefficients $u_{2}$ and $v_{2}$, we immediately have

$$
\left|a_{2}\right|=\frac{|x \sqrt{2 x}| \cos ^{2} \delta}{\sqrt{x^{2} \cos \delta\left[(\sigma-1)(\sigma+2) \phi_{2}^{2}+2(\sigma+2) \phi_{3}\right]-\left(3 x^{2}-1\right)(\sigma+1)^{2} e^{i \delta} \phi_{2}^{2}}},
$$

which easily yields the bound on $\left|\mathrm{a}_{2}\right|$ as asserted in (2.1).

Next, in order to find the bound on $\left|a_{3}\right|$, by subtracting (2.9) from (2.6), we get

$$
e^{i \delta}\left[2(\sigma+2) \phi_{3} a_{3}-2(\sigma+2) \phi_{3} a_{2}^{2}\right]=\left(u_{2}-v_{2}\right) x \cos \delta .
$$

It follows from (2.11), (2.12), and (2.14) that

$$
a_{3}=\frac{\left(u_{2}-v_{2}\right) x e^{-i \delta} \cos \delta}{2(\sigma+2) \phi_{3}}+\frac{\left(u_{1}^{2}+v_{1}^{2}\right) x^{2} e^{-2 i \delta} \cos ^{2} \delta}{2(\sigma+1)^{2} \phi_{2}^{2}} .
$$

Applying Lemma 2.1, the coefficients $u_{1}, v_{1}, u_{2}$, and $v_{2}$, we readily get

$$
\left|a_{3}\right| \leqq \frac{|x| \cos \delta}{(\sigma+2) \phi_{3}}+\left(\frac{x \cos \delta}{(\sigma+1) \phi_{2}}\right)^{2}
$$

Furthermore, from (2.5) and (2.7), (2.10), and (2.14), we find that

$$
\begin{aligned}
a_{4}= & \frac{5 x^{2} u_{1}\left(u_{2}-v_{2}\right) e^{-2 i \delta} \cos ^{2} \delta}{4(\sigma+1)(\sigma+2) \phi_{2} \phi_{3}}+\frac{5 x^{3} u_{1}\left(u_{1}^{2}+v_{1}^{2}\right) e^{-3 i \delta} \cos ^{3} \delta}{4(\sigma+1)^{3} \phi_{2}^{3}} \\
& -\frac{N x^{3} u_{1}^{3} e^{-3 i \delta} \cos ^{3} \delta}{2(\sigma+1)^{3}(\sigma+3) \phi_{2}^{3} \phi_{4}}+\frac{\left(3 x^{2}-1\right)\left(u_{1} u_{2}-v_{1} v_{2}\right) e^{-i \delta} \cos \delta}{2(\sigma+3) \phi_{4}} \\
& +\frac{x\left(u_{3}-v_{3}\right) e^{-i \delta} \cos \delta}{2(\sigma+3) \phi_{4}}+\frac{\left(5 x^{3}-3 x\right)\left(u_{1}^{3}-v_{1}^{3}\right) e^{-i \delta} \cos \delta}{4(\sigma+3) \phi_{4}} .
\end{aligned}
$$

Applying Lemma 2.1 for the coefficients $u_{1}-u_{3}$ and $v_{1}-v_{3}$, we get

$$
\begin{aligned}
\left|a_{4}\right| \leqslant & \frac{5 x^{2} \cos ^{2} \delta}{2(\sigma+1)(\sigma+2) \phi_{2} \phi_{3}}+\frac{5 x^{3} \cos ^{3} \delta}{2(\sigma+1)^{3} \phi_{2}^{3}} \\
& -\frac{N x^{3} \cos ^{3} \delta}{2(\sigma+1)^{3}(\sigma+3) \phi_{2}^{3} \phi_{4}}+\frac{\left(5 x^{3}+6 x^{2}-x-2\right) \cos \delta}{2(\sigma+3) \phi_{4}},
\end{aligned}
$$

where

$$
\mathrm{N}=5(\sigma+3) \phi_{4}+2(\sigma-1)(\sigma+3) \phi_{2} \phi_{3}+\frac{(\sigma-1)(\sigma-2)(\sigma+3)}{3} \phi_{2}^{3}
$$

This completes the proof of Theorem 2.2. 


\section{Fekete-Szegö inequalities}

Due to Zaprawa [43], we will give Fekete-Szegö inequalities $\left|a_{3}-\mu a_{2}^{2}\right|$, where $\mu$ is some real number for the above function classes $\mathcal{M}_{\Sigma}^{\lambda, \alpha, \beta}(\delta, \sigma ; x)$ and it looks like the following.

Theorem 3.1. Let $\mathrm{f}$ is fixed as in (1.1) and $\mathrm{f} \in \mathcal{M}_{\Sigma}^{\lambda, \alpha, \beta}(\delta, \sigma ; x)$, then

$$
\left|a_{3}-\mu a_{2}^{2}\right| \leqslant \begin{cases}\frac{|x| \cos \delta}{(\sigma+2) \phi_{3}}, & \text { if } 0<|h(\mu)|<\frac{1}{2(\sigma+2) \phi_{3}}, \\ 2|x h(\mu)| \cos \delta, & \text { if }|h(\mu)| \geqslant \frac{1}{2(\sigma+2) \phi_{3}},\end{cases}
$$

where $\delta \in\left(-\frac{\pi}{2}, \frac{\pi}{2}\right), \sigma \geqslant 0, \alpha, \beta \in \mathbb{C}, \mathfrak{R}(\alpha)>0, \mathfrak{R}(\beta)>0,0<\lambda \leqslant 1 ; \mu \in \mathbb{R}$ and

$$
h(\mu)=\frac{(1-\mu) x^{2} \cos \delta}{\chi^{2} \cos \delta\left[(\sigma-1)(\sigma+2) \phi_{2}^{2}+2(\sigma+2) \phi_{3}\right]-\left(3 \chi^{2}-1\right)(\sigma+1)^{2} e^{i \delta} \phi_{2}^{2}},
$$

further the coefficients $\phi_{\mathrm{k}}$ are given by (1.5).

Proof. From (2.13) and (2.14), we obtain

$$
\begin{aligned}
& a_{3}-\mu a_{2}^{2}=(1-\mu) \frac{x^{3}\left(u_{2}+v_{2}\right) e^{-i \delta} \cos ^{2} \delta}{\chi^{2} \cos \delta\left[(\sigma-1)(\sigma+2) \phi_{2}^{2}+2(\sigma+2) \phi_{3}\right]-\left(3 x^{2}-1\right)(\sigma+1)^{2} e^{i \delta} \phi_{2}^{2}}+\frac{x\left(u_{2}-v_{2}\right) e^{-i \delta} \cos \delta}{2(\sigma+2) \phi_{3}}, \\
& =\left(\frac{(1-\mu) x^{3} e^{-i \delta} \cos ^{2} \delta}{x^{2} \cos \delta\left[(\sigma-1)(\sigma+2) \phi_{2}^{2}+2(\sigma+2) \phi_{3}\right]-\left(3 x^{2}-1\right)(\sigma+1)^{2} e^{i \delta} \phi_{2}^{2}}+\frac{x e^{-i \delta} \cos \delta}{2(\sigma+2) \phi_{3}}\right) u_{2} \\
& +\left(\frac{(1-\mu) x^{3} e^{-i \delta} \cos ^{2} \delta}{x^{2} \cos \delta\left[(\sigma-1)(\sigma+2) \phi_{2}^{2}+2(\sigma+2) \phi_{3}\right]-\left(3 x^{2}-1\right)(\sigma+1)^{2} e^{i \delta} \phi_{2}^{2}}-\frac{x e^{-i \delta} \cos \delta}{2(\sigma+2) \phi_{3}}\right) v_{2} \text {. }
\end{aligned}
$$

So, we have

$$
a_{3}-\mu a_{2}^{2}=x e^{-i \delta} \cos \delta\left[\left(h(\mu)+\frac{1}{2(\sigma+2) \phi_{3}}\right) u_{2}+\left(h(\mu)-\frac{1}{2(\sigma+2) \phi_{3}}\right) v_{2}\right],
$$

where

$$
h(\mu)=\frac{(1-\mu) x^{2} \cos \delta}{x^{2} \cos \delta\left[(\sigma-1)(\sigma+2) \phi_{2}^{2}+2(\sigma+2) \phi_{3}\right]-\left(3 x^{2}-1\right)(\sigma+1)^{2} e^{i \delta} \phi_{2}^{2}} .
$$

Then, by taking modulus of (3.1), we conclude that

$$
\left|a_{3}-\mu a_{2}^{2}\right| \leqslant\left\{\begin{array}{lll}
\frac{|x| \cos \delta}{(\sigma+2) \phi_{3},}, & \text { if } 0<|h(\mu)|<\frac{1}{2(\sigma+2) \phi_{3}}, \\
2|x h(\mu)| \cos \delta, & \text { if } \quad|h(\mu)| \geqslant \frac{1}{2(\sigma+2) \phi_{3}} .
\end{array}\right.
$$

Taking $\mu=1$, we have the following corollary.

Corollary 3.2. Let $\mathrm{f}$ be assumed in (1.1) and $\mathrm{f} \in \mathcal{M}_{\Sigma}^{\lambda, \alpha, \beta}(\delta, \sigma ; x)$, then

$$
\left|a_{3}-a_{2}^{2}\right| \leqslant \frac{|x| \cos \delta}{(\sigma+2) \phi_{3}}
$$

where $\delta \in\left(-\frac{\pi}{2}, \frac{\pi}{2}\right), \sigma \geqslant 0, \alpha, \beta \in \mathbb{C}, \mathfrak{R}(\alpha)>0, \mathfrak{R}(\beta)>0,0<\lambda \leqslant 1$ and the coefficients $\phi_{\mathrm{k}}$ are given by (1.5). 


\section{Concluding remark}

Various other fascinating consequences of our general results (which are asserted by Theorems 2.2 and 3.1 and Corollaries stated above) can be derived by aptly specializing the parameters $\delta, \sigma$ for the classes $\mathcal{B}_{\Sigma}^{\lambda, \alpha, \beta}(\sigma ; x) ; \mathcal{S}_{\Sigma}^{\lambda, \alpha, \beta}(\delta, \sigma ; \chi)$ and $\mathcal{R}_{\Sigma}^{\lambda, \alpha, \beta}(\delta, \sigma ; x)$ as mentioned in Remark 1.3. The details involved may be left as an exercise for the interested reader who may also consider exploring the problem of further extending the work presented here to hold true for such greatly more convolutions operators.

\section{Acknowledgment}

We authors thank the referees for their valuable suggestion to imrove the paper in present form. Further we would like to thank the Deanship of Scientific Research, Qassim University for funding publication of this project.

\section{References}

[1] F. M. Al-Aboudi, n-Bazilevic functions, Abstr. Appl. Anal., 2012 (2012), 1-10. 1.1

[2] S. Abelman, K. A. Selvakumaran, M. M. Rashidi, S. D. Purohit, Subordination conditions for a class of non-Bazilevič type defined by using fractional q-calculus operators, Facta Univ. Ser. Math. Inform., 32 (2017), 255-267.

[3] A. A. Amer, M. Darus,Distortion theorem for certain class of Bazilevič functions, Int. J. Math. Anal., 6 (2012), 591-597. 1.1

[4] A. Attiya, Some applications of Mittag-Leffler function in the unit Disk, Filomat, 30 (2016), 2075-2081. 1.2

[5] D. Bansal, J. K. Prajapat, Certain geometric properties of the Mittag-Leffler functions, Complex Var. Elliptic Equ., 61 (2016), 338-350. 1.2

[6] D. A. Brannan, J. G. Clunie, Aspects of Contemporary Complex Analysis, Academic Press, London-New York, (1980). 1.1

[7] D. A. Brannan, J. Clunie, W. E. Kirwan, Coefficient estimates for a class of star-like functions, Canadian J. Math., 22 (1970), 476-485.

[8] D. A. Brannan, T. S. Taha, On some classes of bi-univalent functions, Studia Univ. Babeş-Bolyai Math. 31(1986), 70-77. 1.1

[9] M. Çağlar, H. Orhan, N. Yağmur, Coefficient bounds for new subclasses of bi-univalent functions, Filomat, 27 (2013), 1165-1171. 1.1

[10] P.L.Duren Univalent Functions. In: Grundlehren der Mathematischen Wissenschaften, Band 259, New York, Berlin, Heidelberg and Tokyo, Springer-Verlag, 1983. 1.1, 2.1

[11] E. Deniz, Certain subclasses of bi-univalent functions satisfying subordinate conditions, J. Class. Anal., 2 (2013), 49-60. 1.1

[12] A. Ebadian, N. E. Cho, E. A. Adegani, S. Bulut, T. Bulboacă, Radii problems for some classes of analytic functions associated with Legendre polynomials of odd degree, J. Inequal. Appl., 2020 (2020), 12 pages. 1.3

[13] S. M. El-Deeb, B. M. El-Matary, New subclasses of bi-univalent functions connected with aq-analogue of convolution based upon the Legendre polynomials, Stud. Univ. Babeş-Bolyai Math., (accepted for publications-2020). 1.2

[14] B. A. Frasin, An application of an operator associated with generalized Mittag-Leffler function, Konuralp J. Math., 7 (2019), 199-202. 1.2

[15] B. A. Frasin, T. Al-Hawary, F. Yousef, Some properties of a linear operator involving generalized Mittag-Leffler function, Stud. Univ. Babeş-Bolyai Math., 65 (2020), 67-75. 1.2

[16] B. A. Frasin, M. K. Aouf,New subclasses of bi-univalent functions, Appl. Math. Lett., 24 (2011), 1569-1573. 1.1

[17] M. Garg, P. Manohar, S. L. Kalla, A Mittag-Leffler-typefunction of two variables, Integral Transforms Spec. Funct., 24 (2013), 934-944. 1.2

[18] S. P. Goyal, P. Goswami, Estimate for initial Maclaurin coefficients of bi-univalent functions for a class defined by fractional derivatives, J. Egyptian Math. Soc., 20 (2012), 179-182. 1.1

[19] T. Hayami, S. Owa, Coefficient bounds for bi-univalent functions, PanAmer. Math. J., 22 (2012), 15-26. 1.1

[20] Y. C. Kim, H. M. Srivastava, The Hardy space for a certain subclass of Bazilevič functions, Appl. Math. Comput., 183 (2006), 1201-1207. 1.1

[21] Y. C. Kim, T. Sugawa, A note on Bazilevič functions, Taiwanese J. Math., 13 (2009), 1489-1495. 1.1

[22] V. Kiryakova, Generalized Fractional Calculus and Applications, Longman Scientific \& Technical, Harlow; copublished in the United States with John Wiley \& Sons, New York, (1994). 1.2

[23] N. N. Lebedev, Special functions and their applications, Dover Publications, New York, (1972). 1.3

[24] M. Lewin, On a coefficient problem for bi-univalent functions, Proc. Amer. Math. Soc., 18 (1967), 63-68. 1.1

[25] X.-F. Li, A.-P. Wang, Two new subclasses of bi-univalent functions, Int. Math. Forum, 7 (2012), 1495-1504. 1.1 
[26] W. C. Ma, D. Minda, A unified treatment of some special classes of functions, In: Conf. Proc. Lecture Notes Anal., I, Int. Press, Cambridge, MA, (1994), 157-169. 1

[27] S. S. Miller, P. T. Mocanu, Differential Subordination: Theory and Applications, CRC Press, New York and Basel, (2000). 1

[28] G. M. Mittag-Leffler, Sur la nouvelle fonction E(x), C. R. Acad. Sci. Paris, 137 (1903), 554-558. 1.2

[29] G. Murugusundaramoorthy, S. M. El-Deeb, Second Hankel determinant for a class of analytic functions of the MittagLeffler-type Borel distribution related with Legendre polynomials, Turkish World Math. Soc. J. Appl. Engineering Math., (accepted for publications-2021). 1.2, 1.2

[30] G. Murugusundaramoorthy, T. Janani, S. D. Purohit, Coefficient estimate of bi-Bazilevič functions associated with fractional q-calculus operators, Fund. Inform., 151 (2017), 49-62. 1.1

[31] E. Netanyahu, The minimal distance of the image boundary from the origin and the second coefficient of a univalent function in $z<1$, Arch. Rational Mech. Anal., 32 (1969), 100-112. 1.1

[32] K. I. Noor, K. Ahmad, On higher order Bazilevic functions, Internat. J. Modern Phys. B, 27 (2013), 14 pages. 1.1

[33] K. A. Selvakumaran, H. A. AL-Kharsani, D. Baleanu, S. D. Purohi, K. S. Nisar, Inclusion relationships for some subclasses of analytic functions associated with generalized Bessel functions, J. Comput. Anal. Appl., 24 (2018), 81-90. 1.2

[34] C. Selvaraj, C. S. Moni, Subordination results for a class of Bazilević functions with respect to symmetric points, Stud. Univ. Babeş-Bolyai Math., 58 (2013), 23-30. 1.1

[35] R. Singh, On Bazilevič functions, Proc. Amer. Math. Soc., 38 (1973), 261-271. 1.1

[36] H. M. Srivastava, S. Bulut, M. Çağlar, N. Yağmur, Coefficient estimates for a general subclass of analytic and bi-univalent functions, Filomat 27 (2013), 831-842. 1.1

[37] H. M. Srivastava, A. K. Mishra, P. Gochhayat, Certain subclasses of analytic and bi-univalent functions, Appl. Math. Lett., 23 (2010), 1188-1192. 1.1

[38] H. M. Srivastava, G. Murugusundaramoorthy, K. Vijaya, Coefficient estimates for some families of bi-Bazilevič functions of the Ma-Minda type involving the Hohlov operator, J. Class. Anal., 2 (2013), 167-181. 1.1, 1.3

[39] T. S. Taha, Topics in Univalent Function Theory, Ph.D. Thesis, University of London, (1981). 1.1

[40] A. K. Wanas, J. A. Khuttar, Applications of Borel distribution series on analytic functions, Earthline J. Math. Sci., 4 (2020), 71-82. 1.2

[41] Z.-G. Wang, H.-T. Wang, Y. Sun, A class of multivalent non-Bazilevič functions involving the Cho-Kwon-Srivastava operator, Tamsui Oxf. J. Math. Sci., 26 (2010), 1-19. 1.1

[42] A. Wiman, Über die Nullstellen der Funktionen E ${ }^{\mathrm{a}}(\mathrm{x})$, Acta Math., 29 (1905), 217-134. 1.2

[43] P. Zaprawa, On the Fekete-Szegö problem for classes of bi-univalent functions, Bull. Belg. Math. Soc. Simon Stevin, 21 (2014), 169-178. 3 